\title{
DYNAMIC OBSERVATION OF DOMAIN SHRINKING SPEED OVER WALKER'S LIMIT
}

\author{
F. Izawa, S. Ozawa, A. Tsukamoto, K. Nakagawa, and A. Itoh \\ Dept. Electronics and Computer Science, College of Science \& Technology, \\ Nihon University, Funabashi, 274-8501 Japan
}

When measuring the domain shrinking velocity of $\mathrm{GdFeCo}$ single layer film, which is an expanding layer of Magnetic AMplifying MO System (MAMMOS) ${ }^{1)}$, scattering was seen in the shrinking velocity when the effective driving field was over 100 Oe. Walker's limit was thought of as the cause of the scattering, and then the damping constant was determined to be 0.29 . When the in-plane field was applied, the scattering disappeared, and an increase in shrinking velocity was seen.

Key words: magnetic amplifying $\mathrm{MO}$ system (MAMMOS), GdFeCo, wall coercivity, Walker's break down, damping constant, wall velocity, Bloch wall, in-plane field,

\section{Introduction}

In MAMMOS, when a small domain in the recording layer is copied to the readout layer, the domain expands to an enhanced readout signal. After the expansion, the domain should be collapsed for reading out the next information. The velocity of the expansion and the shrinking of the domain raises as an important parameter which determines the readout speed of MAMMOS. The shrinking speed was derived by dynamic observations of the shrinking processes under the pulse magnetic field for shrinking. We already reported ${ }^{2)}$ the method and the dynamic observation results of the film used for the MAMMOS readout layer. In this report Walker's critical field $\mathrm{H}_{\mathrm{cr}}$ and the dumping constant $\alpha$ were derived from the dynamic observation results.

We also showed by experiment, when the in-plane field is applied, the wall velocity becomes proportional to the effective driving field, and is it faster than without an in-plane field, even after Walker's break down takes place.

\section{Experimental method}

We have developed ${ }^{2)}$ a stroboscopic observation system for diagnosing the dyanamic domain phenomena. This system and the time chart of magnetic fields, a writing laser pulse, an observing laser pulse, and an electric shutter -- in order to measure the domain shrinking speed -- are almost the same as reported before ${ }^{2)}$, except a newly developed coil driver circuit applied a higher shrinking field than before.

The film structure is: glass substrate, $100 \mathrm{~nm} \mathrm{SiN}, 40 \mathrm{~nm}$ $\mathrm{GdFeCo}$, and a $100 \mathrm{~nm} \mathrm{SiN}$ cover layer. Magnetization Ms and coercivity $\mathrm{Hc}$ at room temperature are $55 \mathrm{emu} / \mathrm{cc}$ and 210 Oe, respectively.

\section{Experimental Results}

\subsection{Static measurement of wall coercivity $\mathbf{H}_{w}$}

The effective field $\mathrm{H}_{\text {eff }}$ for shrinking the written domain is $\mathrm{H}_{\text {eff }}=\mathrm{H}_{\mathrm{a}}-\mathrm{H}_{\mathrm{w}}$, where $\mathrm{H}_{\mathrm{a}}$ is the applied field and $\mathrm{H}_{\mathrm{w}}$ is the wall coercivity. $\mathrm{H}_{\mathrm{w}}$ was estimated by measuring the external bias field $\mathrm{H}_{1}$ and $\mathrm{H}_{2} . \mathrm{H}_{1}$ initiates the expansion and $\mathrm{H}_{2}$ the value of the field at the onset of the collapse of written circular domains. $\mathrm{H}_{\mathrm{w}}$ was derived as follows.

$$
H_{W}=\left|H_{1}+H_{2}\right| / 2
$$

This static measurement method captures ambiguities in the expansion and collapse of domains, so the measurements were performed many times to determine $\mathrm{H}_{\mathrm{w}}$. As a consequence, $\mathrm{H}_{\mathrm{w}}$ was estimated as $16 \mathrm{Oe}$.

\subsection{Case without an in-plane field}

Walker ${ }^{3)}$ carried out analysis on the perturbation of a domain wall, including an exact solution of the spin distribution throughout a wall moving in response to an applied magnetic field. According to Walker's analysis for the motion of the infinite plane Bloch wall, the critical field $\mathrm{H}_{\mathrm{cr}}$ is shown as equation (2).

$$
H_{c r}=2 \pi \alpha M_{s}
$$

$\alpha$ is the damping constant. When $\mathrm{H}_{\text {eff }}$ is lower than $\mathrm{H}_{\mathrm{cr}}$, magnetic moments rotate all together. Thus, the domain wall moves without breaking its structure. But, when $\mathrm{H}_{\text {eff }}$ is higher than $\mathrm{H}_{\mathrm{cr}}$, they rotate separately, and the domain wall moves until breaking its structure. In the former, the

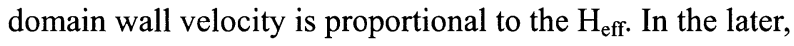
the domain wall velocity is unrelated to the $\mathrm{H}_{\mathrm{eff}}$.

We already reported ${ }^{2)}$ on the domain shrinking speed of only up to 110 Oe with the velocity rising to $80 \mathrm{~m} / \mathrm{sec}$. In this report, the maximum applied field is $160 \mathrm{Oe}$.

The domain shrinking speed, estimated from the dynamic observations of domain shapes during shrinking, is up to $160 \mathrm{Oe}$, as shown in Fig. 1 as a function of the effective driving field $\mathrm{H}_{\mathrm{eff}}$.

The domain wall velocity is proportional $\mathrm{H}_{\text {eff }}$ up to 94 Oe. But when $\mathrm{H}_{\text {eff }}$ equals more than around 99 Oe, the wall velocity scatters widely on every measurement. 


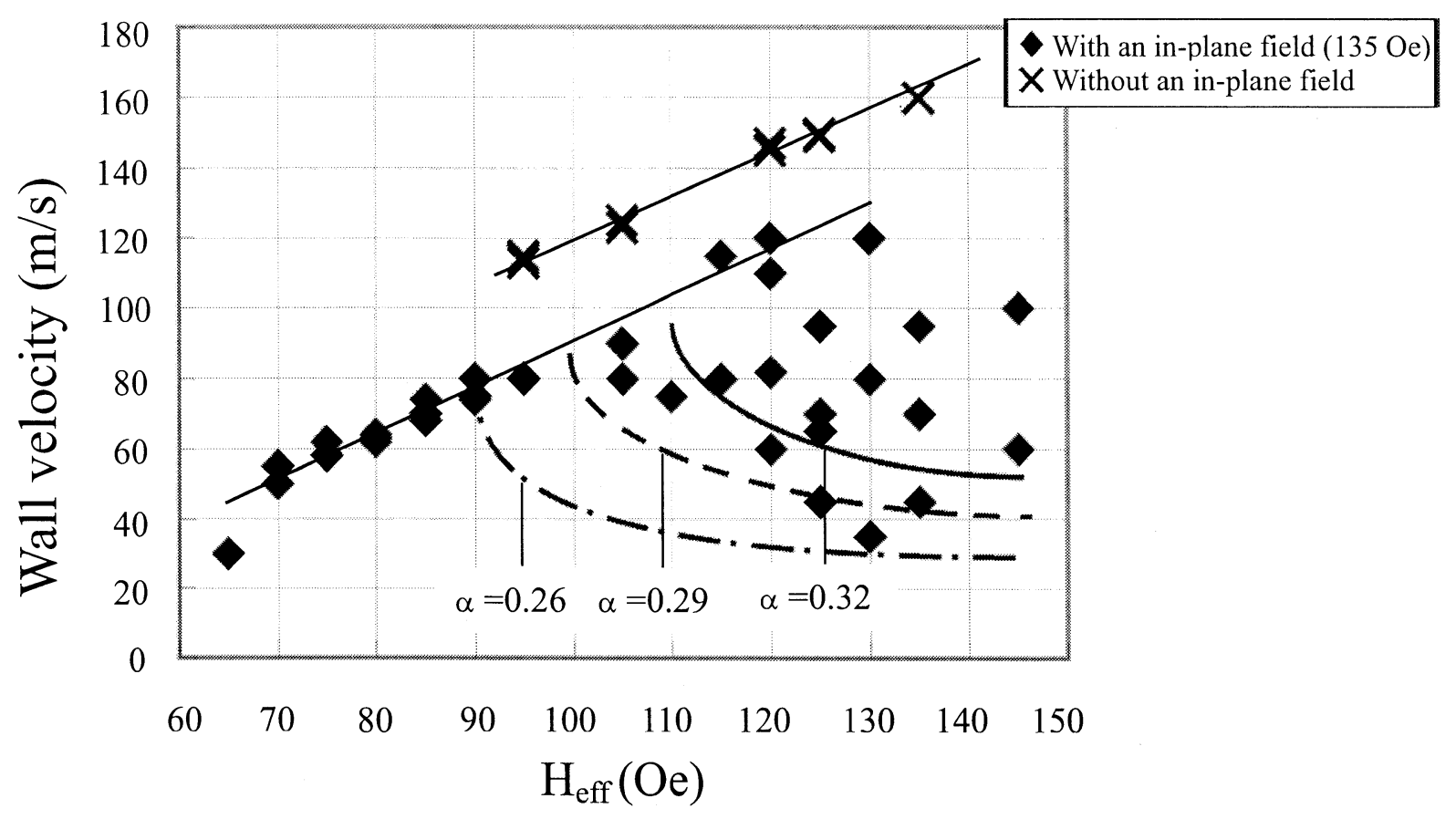

Fig. 1 Wall velocity as function of the effective driving field $\mathrm{H}_{\text {eff }}$ with and without an in-plane field. Theoretical curves after Walker's break down are shown with the solid line $(\alpha=0.32)$, and the dashed line $(\alpha=0.29)$, respectively. The velocity of the in-plane field is shown by crosses and faint line.

The reason $\mathrm{H}_{\text {eff }}$ approaches the critical field is shown by Walker's formula in Eq, (2).

The wall velocity after Walker's break down is shown in equation (3) ${ }^{4)}$ below, where $\gamma$ is gyromagnetic ratio and $\Delta$ is wall width parameter.

$$
v=\frac{2 \pi \gamma \Delta \mathrm{M}_{\mathrm{S}}}{1+\alpha^{2}}\left(\alpha^{2} h+\frac{1}{h+\sqrt{h^{2}-1}}\right)
$$

Where $h$ and $\Delta$ are defined as follows.

$$
\begin{aligned}
h & =\frac{H_{e f f}}{2 \pi \alpha M_{S}} \\
\Delta & =\sqrt{\frac{A}{K u}}
\end{aligned}
$$

In order to derive damping constant $\alpha$, we fit the estimated velocity as a function of the effective field. Because the wall velocity became unstable at $\mathrm{H}_{\mathrm{cr}}=99 \mathrm{Oe}$, we set equation (3) at $\alpha=0.29$ with the experimental results in Fig. 1. It is also shown in Fig. 1 as a dashed line. The same process was done at $\mathrm{H}_{\mathrm{cr}}=90$, and $110 \mathrm{Oe}$. Derived $\alpha$ were 0.26 and 0.32 , respectively. They are also shown in Fig. 1. Among them, $\alpha=0.29$ is seemed to be well-fit.

In the case of $h=1\left(\mathrm{H}_{\mathrm{eff}}=\mathrm{H}_{\mathrm{cr}}\right)$, Eq. (3) is

$$
v_{c r}=2 \pi \gamma \Delta M_{S}
$$

The value of $\Delta$ estimated from $v_{\mathrm{cr}}=80 \mathrm{~m} / \mathrm{s}, \mathrm{M}_{\mathrm{s}}=55$ $\mathrm{emu} / \mathrm{cc}$ and $\gamma=1.76 \times 10^{7} \mathrm{rad} / \mathrm{s} \cdot \mathrm{Oe}$ is $13.2 \mathrm{~nm}$.

\subsection{Case with an in-plane field}

$\mathrm{N}$. Hayashi et $\mathrm{al}^{5)}$ reported the effect of the external in-plane field on the copying and the expansion of the domain in the readout layer of MAMMOS by solving the LLG equation numerically.

They found, in the one-dimensional simulation, the three kinds of wall structures exist depending on the magnitude of the in-plane field, Bloch wall, Neel-like wall, or a mixture of those. The existence of the in-plane field in the readout layer not only affects the structure of the wall, but also tends to increase the wall velocity and the range of $\mathrm{H}_{\mathrm{eff}}$ for steady-state wall motion.

We performed the experiments for measuring the shrinking speed of domains under the in-plane field $\mathrm{H}_{\text {para }}$. $\mathrm{H}_{\text {para }}$ is applied with a permanent magnet. Fig. 1 also shows the domain shrinking speed as a function of $\mathrm{H}_{\text {eff }}$ with the in-plane field.

When an in-plane field is applied, the wall velocity becomes proportional to the effective driving field faster than without an in-plane field, even after Walker's break 
down. The experiments also showed that applying a 135 Oe in-plane field makes the domain shrinking speed $43 \%$ faster than without it.

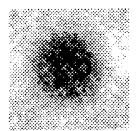

$\mathrm{t}=0 \mathrm{nsec}$

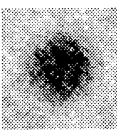

19

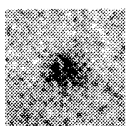

26

27
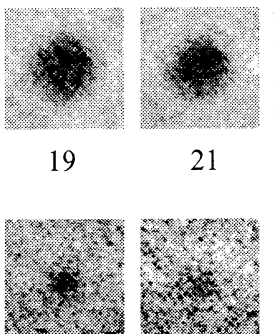

21
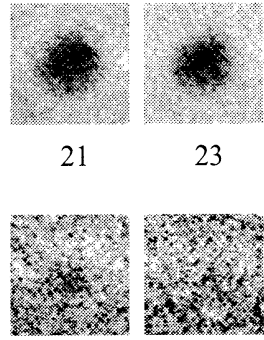

28
23

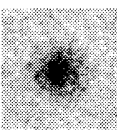

25

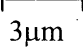

29 (none) (a) $\mathrm{H}_{\text {eff }}=94 \mathrm{Oe}, \mathrm{H}_{\text {para }}=0 \mathrm{Oe}$

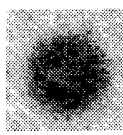

$\mathrm{t}=0$ nsec

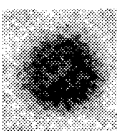

18

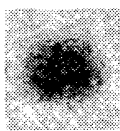

21

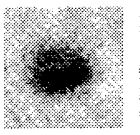

22

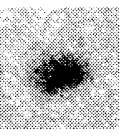

23
Direction of in-plane field

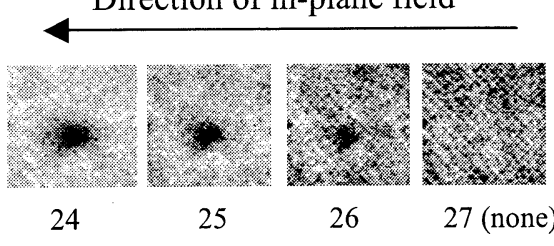

(b) $\mathrm{H}_{\text {eff }}=94 \mathrm{Oe}, \mathrm{H}_{\mathrm{para}}=135 \mathrm{Oe}$

Fig. 2 Example of observed domain shrinking processes at room temperature. (a): $\mathrm{H}_{\text {eff }}=94 \mathrm{Oe}, \mathrm{H}_{\text {para }}$ $=0 \mathrm{Oe}$, without an in-plane field. (b): $\mathrm{H}_{\mathrm{eff}}=94 \mathrm{Oe}$ and $\mathrm{H}_{\text {para }}=135 \mathrm{Oe}$.

In Fig. 2, examples of observed domain shrinking processes at $\mathrm{H}_{\text {eff }}=94 \mathrm{Oe}$, both without and with an in-plane field, are shown. When an in-plane field was applied, the shapes of the domain during the shrinking process were different from that without an in-plane field. The direction of the applied in-plane field is also shown in Fig. 2. The change of domain diameters during shrinking processes were measured by using the Fig. 2(a) and (b). Fig. 2(b) shows that there was a field applied in-plane, which causes the domain to form an elliptic. Here the major and minor axes were measured.

In Fig. 3, the domain diameter as a function of time in the shrinking process is shown for the cases without a field applied in-plane, and with a field applied in-plane. In the latter, the major and minor axes and their average are plotted as functions of time.

We estimated the domain shrinking speed from the

gradient of domain diameter as a function of time in Fig. 3 Where there was no field applied, the domain shrinking speed is $80 \mathrm{~m} / \mathrm{s}$. Where there was a field applied in-plane, it was $110 \mathrm{~m} / \mathrm{s}$. These experiments showed 1350e of an in-plane field makes the domain shrinking speed $43 \%$ faster than without an in-plane field. Moreover, in Fig. 1, an in-plane field suppresses the scattering of wall velocity over Walker's break down.

When there was no in-plane field, the circular domain shrank keeping its shape. When there was an in-plane field, the circular domain once changed into an ellipse, then it became circular again, and disappeared. In the former case, the ratio of the major and minor axes is less than 1.05 during the shrinking process. However, in the later case, the ratio increased from 1.0 at the beginning to 1.5 in the middle, and then decreased to 1.0 just before collapsing. The major axis of the elliptical shape of domains was aligned parallel to the in-plane field. Both ends of the elliptical domains on the major axis moved slower than the others. So an in-plane field not only affects the shrinking speed, but also affects the pinning field.

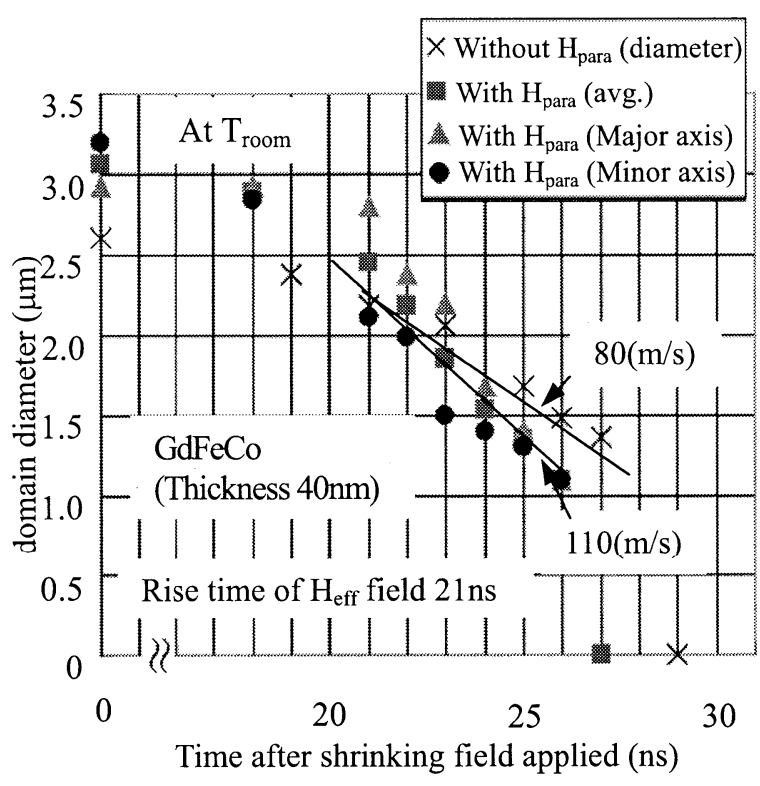

Fig. 3 Time dependence of domain shape during shrinking. Without an in-plane field $\left(\mathrm{H}_{\mathrm{para}}=0\right)$, the diameter of the domain is plotted. With an in-plane field $\left(\mathrm{H}_{\mathrm{para}}=135 \mathrm{Oe}\right)$, major and minor axes and also the average value of those two are plotted.

\section{Conclusions}

We performed dynamic observations of domain shrinking processes in GdFeCo film for a MAMMOS expanding layer over a wide range of applied fields 
covering the range over Walker's break down. As a result, the domain wall velocity is proportional to the effective driving field $\mathrm{H}_{\text {eff }}$ up to $94 \mathrm{Oe}$. But when the $\mathrm{H}_{\text {eff }}$ equals more than around $99 \mathrm{Oe}$, the wall velocity scatters widely on every measurement. And the value of damping factor $\alpha$ is estimated at 0.29 .

A 135 Oe in-plane field makes the domain shrinking speed $43 \%$ faster than without an in-plane field, but due to the in-plane field the domains change to an elliptic shape because their ends are somehow pinned during shrinking. An in-plane field not only tends to increase wall velocity, but also suppresses the scattering of the wall velocity after Walker's break down.

\section{References}

1) H. Awano, H. Shirai, and N, Ohta: SPIE Vol. 310, 83-86 (1997).

2) A. Itoh, M. Akiyama, T. Ishikawa, and K. Nakagawa: Trans. Magn. Soc. Jpn., No4-2, 258-263 (2002).

3) N. L. Schrer and L. R. Walker: J. R. Walker: J. Appl. Phys., 45, 5406 (1974)

4) H. C. Bourne, Jr. and D. S. Bartram: IEEE Trans. Magm. MAG-8, 741 (1972).

5) N. Hayashi, Y. Nakatani, H. Awano, and N. Ohta: J. Magn. Soc. Jpn., 23(Supple. No. S1), pp.151-156 (1999).

Received April 16, 2004; Accepted July 5, 2004. 